\title{
Paroxysmal nocturnal hemoglobinuria: role of the complement system, pathogenesis, and pathophysiology
}

\author{
Meryem Bektas, PhD, MBA; Catherine Copley-Merriman, MS, MBA; Shahnaz Khan, MPH; \\ Sujata P Sarda, BPharm, PhD; and Jamile M Shammo, MD, FASCP, FACP
}

\section{SUMMARY}

The complement system is part of the innate immune response system, which comprises more than 50 distinct plasma and serum proteins that interact to opsonize pathogens (i.e., mark pathogens for destruction) and induce inflammatory responses to fight infection. The role of the complement system is 2-fold: immune surveillance and host defense. Paroxysmal nocturnal hemoglobinuria (PNH) is a rare, chronic, acquired, hematologic disease caused by somatic mutations in the gene PIGA in the hematopoietic stem cells. These stem cells produce abnormal clone blood cells that lack the complement regulatory proteins CD55 and CD59, causing the body to recognize these otherwise healthy red blood cells as damaged. The complement system destroys cells without these protective proteins, resulting in general hemolysis. $\mathrm{PNH}$ is characterized by fatigue; hemolytic anemia that can be severe and debilitating; increased lactic dehydrogenase level, reticulocyte count, and bilirubin level; propensity for thrombotic events; and renal dysfunction. Epidemiologic data, while sparse, suggest that an estimated 5,000-6,000 individuals in the United States are affected by PNH. If left untreated, $\mathrm{PNH}$ has a 10 -year mortality rate of $29 \%$, although the natural history of this disease has been recently altered by the introduction of complement inhibitors for the treatment of $\mathrm{PNH}$.

\section{Author affiliations \\ Meryem Bektas, PhD, MBA; Catherine Copley-Merriman, MS, MBA; and Shahnaz Khan, MPH, Market Access and Outcomes Strategy, RTI Health Solutions, Research Triangle, NC. Sujata P Sarda, BPharm, PhD, Global Health Economics and Outcomes Research, Apellis Pharmaceuticals, Waltham, MA, and Jamile M Shammo, MD, FASCP, FACP, Department of Internal Medicine, Rush University Medical Center, Chicago, IL.}

AUTHOR CORRESPONDENCE:

Meryem Bektas, 919.541.6776;

mbektas@rti.org

$J$ Manag Care Spec Pharm 2020;26(12-b):S3-S8

Copyright $\odot 2020$, Academy of Managed Care Pharmacy. All rights reserved.

\section{Overview of the Complement System}

The complement system plays an important role in mediating the hemolysis that characterizes paroxysmal nocturnal hemoglobinuria (PNH). The complement system is part of the innate immune response system, composed of more than 50 distinct plasma and serum proteins that interact to opsonize pathogens and induce inflammatory responses to fight infection. Opsonization is the process of marking pathogens for destruction by recognizing and targeting invading particles for phagocytosis by coating them with antibodies or complement components. ${ }^{1,2}$ There are 3 distinct pathways through which the complement cascade can be activated on different molecules for their initiation (classical, lectin, and alternative), but these pathways converge at the point of $\mathrm{C} 3$ in the cascade to generate the same set of effector molecules. ${ }^{1,3}$ The classical pathway is triggered by antibody or direct binding of the complement component $\mathrm{Clq}$ to the pathogen surface; the lectin pathway is triggered by mannanbinding lectin, a normal serum constituent that binds some encapsulated bacteria; and the alternative pathway, which is continuously activated at a low level, is triggered directly on pathogen surfaces.

Small complement proteins circulate in the blood as inactive precursors, waiting to be stimulated by triggers. ${ }^{4}$ 


\section{FIGURE 1 The Complement System}

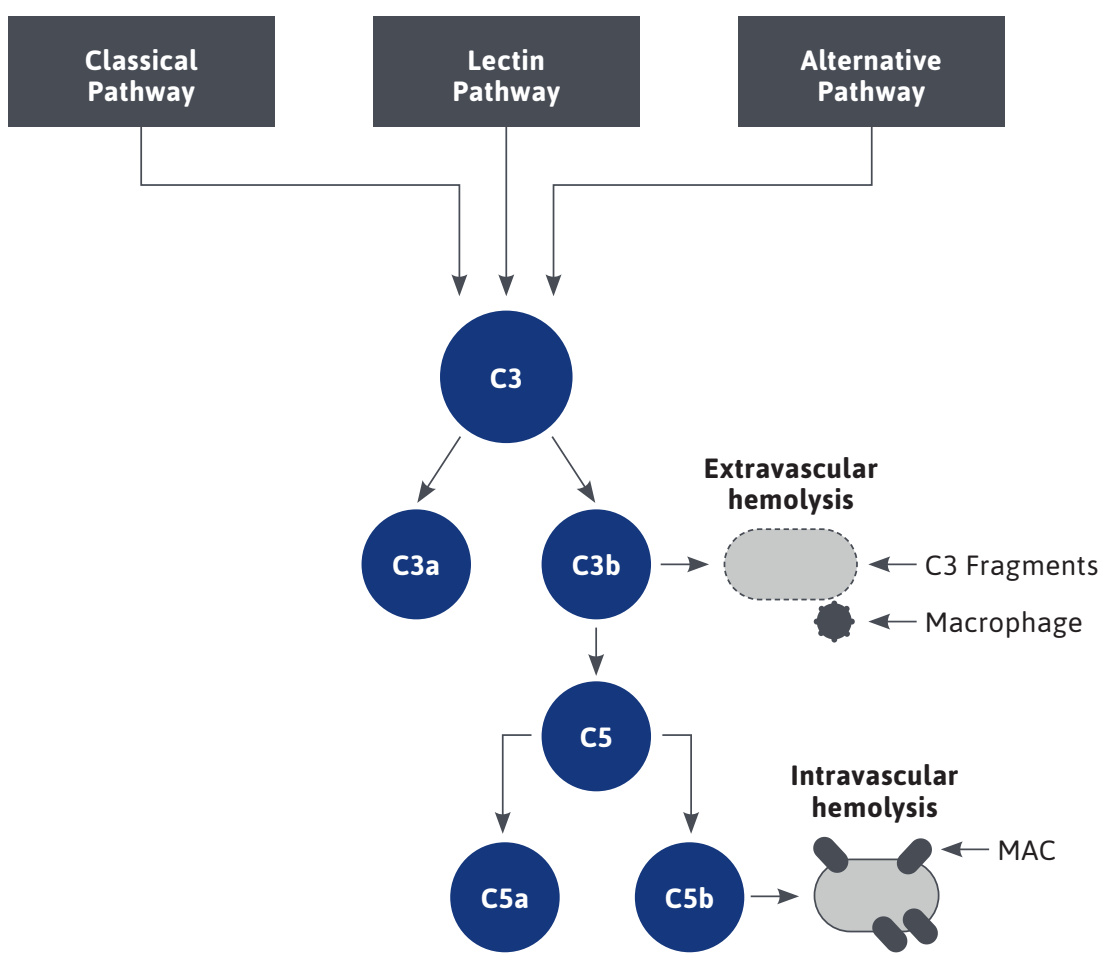

$M A C=$ membrane attack complex.

After a trigger, a complement protein is cleaved into its active form, which then cleaves other proteins into their active forms in a cascade-like manner (Figure 1). ${ }^{4}$ Once fully activated, the complement system culminates in proteolytic, inflammatory, and lytic processes.

In PNH, mutations in the PIGA (phosphatidylinositol glycan A) gene cause the production of abnormal clone red blood cells (RBCs) that lack the complement regulatory proteins CD59 (membrane inhibitor of reactive lysis, MIRL) and CD55 (decay accelerating factor, DAF). ${ }^{5}$ The body recognizes these CD55- and CD59lacking but otherwise healthy RBCs as damaged, and the complement system destroys the cells, resulting in hemolysis. $^{5}$

\section{ROLE OF THE COMPLEMENT CASCADE}

The role of the complement cascade is 2-fold: immune surveillance and host defense. ${ }^{1}$ Defensive effects of the complement cascade are to tag or mark pathogens (opsonization) with activated complement proteins for removal by phagocytes, to induce a series of inflammatory responses that help to fight infection, and to recruit and activate more phagocytes to the site of complement activation, and to damage bacteria by creating pores in the bacterial membrane (lysis; Figure 2). ${ }^{1}$ Complement activity must be regulated to confine the process to invading pathogens and prevent damage to healthy host tissues. ${ }^{3}$ Generation of potent effectors should be tightly regulated, and many steps in the complement activation are checked by regulatory proteins and inhibitors.

A complement-initiated membrane attack complex (MAC) is assembled through the association of several complement proteins. The host cell protein CD59 (MIRL) on the cell surface prevents the aggregation of the complement factor C9 and, hence, the lytic pores. ${ }^{6}$ CD55 (DAF) leads to the destruction of the enzyme C3-convertase, an enzyme that leads to the activation of complement factors $\mathrm{C} 3$ and $\mathrm{C} 5$, both necessary in the MAC. Complement regulatory proteins such as CD55 and CD59 on the surface of the host cells inhibit formation of the $\mathrm{C} 3$ convertases and prevent formation of MAC, respectively, thereby preventing self-targeted complement activation. ${ }^{1}$

\section{RED BLOOD CELLS AND THE COMPLEMENT CASCADE}

Aside from pathogens and damaged host cells, RBCs are potentially sensitive to lysis because they do not have a nucleus. ${ }^{5}$ In RBCs, CD59 and CD55 are cell-surface proteins that are part of the host's self-recognition mechanism; this mechanism protects the cells from being destroyed if the complement system is activated to fight an infection. $^{\text {? }}$

\section{Overview of PNH}

PNH is a rare, chronic, acquired (i.e., not inherited), somatic mutation of the PIGA gene. The mutation occurs randomly and sporadically. In $\mathrm{PNH}$, this mutation occurs in a single hematopoietic stem cell, which then multiplies and expands, manifesting with episodic complement-mediated hemolysis that can result in life-threatening complications, including anemia and venous thrombosis. The classic presentation of $\mathrm{PNH}$ is characterized by sudden, episodic (paroxysmal), at 


\section{FIGURE 2 Defensive Process of the Complement Cascade}

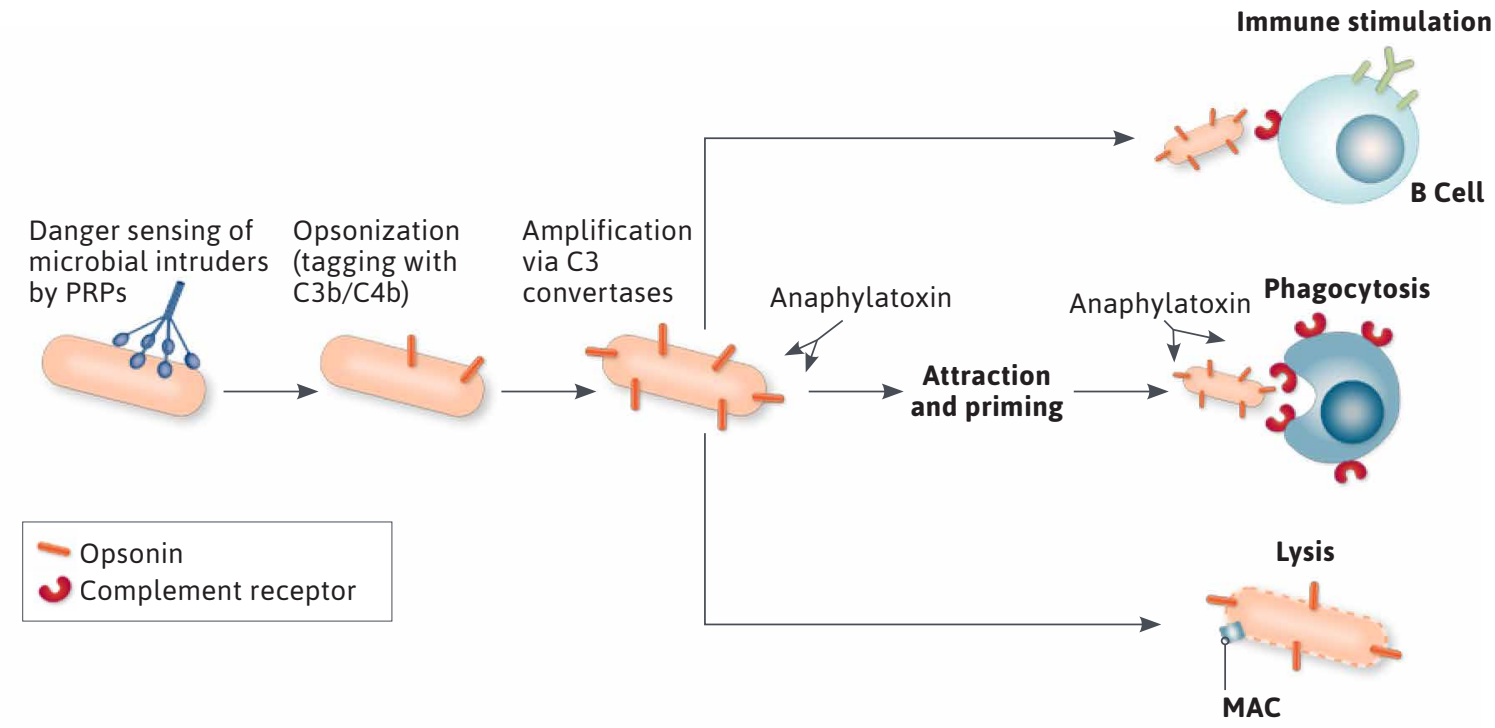

Adapted from: Ricklin D, Mastellos DC, Reis ES, Lambris JD. The renaissance of complement therapeutics. Nat Rev Nephrol. ${ }^{32}$

Danger sensing by PRPs leads to opsonization of the microorganisms with C3b and/or C4b. Opsonization is then amplified via C3 convertases, leading to the initiation of various effector functions including immune stimulation, phagocytosis, and lysis. ${ }^{32}$

$M A C=$ membrane attack complex; $P R P=$ pattern-recognition proteins.

night (nocturnal) hemoglobin occurring in the urine (hemoglobinuria, passing of breakdown product of RBCs into urine). ${ }^{8}$ However, hemoglobin in the urine may not always be visible to the eye, and not every patient with PNH presents with frank hemoglobinuria. While the specific symptoms of $\mathrm{PNH}$ vary from one person to another, the most common symptom of $\mathrm{PNH}$ is debilitating fatigue. ${ }^{9}$

\section{CLASSIFICATION}

Classification of PNH is not well defined, and the disorder is difficult to diagnose. While no universally accepted classification system is available for $\mathrm{PNH}$, a classification scheme has been proposed by the International PNH Interest Group (IPIG) and the PNH International Registry.,10 The classification of PNH subtype helps to categorize the clinical manifestations and severity of hemolysis, and it informs immunological treatment approaches. The IPIG developed a 3-subcategory classification method for PNH that incorporates variations in the presenting features, clinical manifestations, and natural history among patients. ${ }^{7,10}$ Although IPIG has suggested this classification scheme, distinction between the 3 categories may be difficult since varying degrees of bone marrow failure underlie almost all cases of $\mathrm{PNH}^{10}$
Classic or clinical PNH is characterized by significant intravascular hemolytic anemia with no signs of bone marrow failure, an elevated reticulocyte count, high lactic dehydrogenase (LDH) levels, and large PNH clones ( $>50 \%$ of glycosylphosphatidylinositol-anchored protein [GPI-AP]deficient granulocytes). $\mathrm{PNH}$ in the setting of a bone marrow disorder is defined by a history of associated bone marrow disease, karyotypic abnormalities typical of bone marrow disorders (i.e., aplastic anemia or myelodysplastic syndrome [MDS]), and $<50 \%$ of GPI-AP-deficient granulocytes. Conversely, subclinical PNH is asymptomatic, with no clinical or laboratory evidence of hemolysis and $<10 \%$ of GPI-AP-deficient granulocytes.

\section{EPIDEMIOLOGY AND RISK FACTORS}

PNH can affect any age group, with a median age of diagnosis in the $30 \mathrm{~s}$, and is estimated to affect males and females equally. ${ }^{11-13}$ Aplastic anemia is a known risk factor for PNH. In patients with aplastic anemia, the risk of developing clinical $\mathrm{PNH}$ is $15 \%-25 \%{ }^{13}$ If left untreated, 10-year mortality rates among patients with $\mathrm{PNH}$ are $29 \%{ }^{14}$

Because of the rarity of $\mathrm{PNH}$, the incidence and prevalence rates have been poorly reported and have likely been underestimated. ${ }^{12,15}$ However, for stakeholders to best 
estimate how many patients may have the condition, 2 available studies, which include 1 model and 1 known epidemiological study, are summarized. Given the paucity of U.S. epidemiological data, researchers have attempted to model the U.S. incidence and prevalence of PNH. In a study of the evolutionary dynamics of $\mathrm{PNH}$, the prevalence of clinical PNH cases, both monoclonal and multiclonal (i.e., reflecting multiple independently arising clones within the hematopoietic stem cell pool), was estimated in the United States. Using the stochastic evolutionary model and Markov chain model and population age distribution data obtained in 2010 by the U.S. Census Bureau, the researchers estimated that prevalence of clinical PNH for U.S. monoclonal and multiclonal cases was 1.76 cases per 100,000 individuals. For the U.S. population, this would result in an estimate of approximately 3,000 patients with a single clone and 2 patients with biclonal disease based on historical (2010) U.S. population data. ${ }^{16}$

To better define the incidence and prevalence of $\mathrm{PNH}$ in the United Kingdom, researchers collected survival data on all patients diagnosed with $\mathrm{PNH}$ in the strategic health authorities of North and East Yorkshire, Northern Lincolnshire, and West Yorkshire between January 1991 and July 2006. ${ }^{17}$ Seventy-six patients with PNH were diagnosed during this time period, resulting in an annual incidence of 0.13 per 100,000 . Based on this incidence rate and additional data on survival, the estimated 15-year prevalence of $\mathrm{PNH}$ in this study was 1.59 per 100,000 , resulting in a predicted prevalence of 59 patients in the study region. The estimate for the United States based on the same incidence and prevalence estimates and the same assumption about disease occurrence and survival was 4,713 cases of PNH based on the July 1, 2005, U.S. Census Bureau population estimate of 296,410,404. ${ }^{17}$ Based on 2020 U.S. Census Bureau population data, the number of U.S. PNH cases estimated from these United Kingdom data is likely to be 5,000-6,000.17,18

In summary, the 2 known epidemiologically related references containing data about the prevalence of $\mathrm{PNH}$ provide an estimate that the number of U.S. patients ranges between 3,000 and 6,000 per $100,000,{ }^{16,17}$ which can be used by payers as they assess prevalence in their managed care populations.

\section{PATHOPHYSIOLOGY AND SYMPTOMS}

While the specific symptoms, progression, and severity of PNH vary from one person to another, chronic hemolysis is central to the symptoms and clinical manifestations of the disorder. ${ }^{7}$ Fatigue is the most commonly reported symptom of $\mathrm{PNH}$, occurring in $80.9 \%$ of patients. ${ }^{9}$ Other common symptoms include hemoglobinuria (45.0\%), dyspnea (45.3\%), renal dysfunction (42.8\%), abdominal pain (35.2\%), erectile dysfunction (24.2\%), dysphagia (16.5\%), and thrombosis $(13.3 \%) .{ }^{9}$ If left untreated, patients with $\mathrm{PNH}$ experience major adverse vascular events (18.8\%), thrombotic events (13.3\%), and impaired renal function (42.8\%) and must undergo RBC transfusions (61.3\%). ${ }^{9}$ Hemoglobinuria, thrombosis, severe anemia, and debilitating fatigue have a significant effect on daily living due to loss of independence, decreased physical activity, and functional decline..$^{9,19,20}$

In patients with $\mathrm{PNH}$, hemolysis is chronic but may worsen during periods of infection, trauma, or stress. ${ }^{10}$ Hemolysis can occur intravascularly (i.e., within circulation), releasing cellular content into the surrounding environment and leading to an increase in serum LDH level, which can be up to 10 times the upper limit of normal. ${ }^{21}$ Extravascular lysis occurs outside of circulation in the spleen or liver due to RBC opsonization. Extravascular hemolysis is caused by C3d deposition on the PNH red cells. ${ }^{6}$ $\mathrm{PNH}$ results in abnormal hematologic parameters, including decreased hemoglobin and increased $\mathrm{LDH}$, reticulocyte, and bilirubin levels. ${ }^{6,22}$ The premature destruction of RBCs in patients with $\mathrm{PNH}$ may result in hemolytic anemia (i.e., low levels of circulating RBCs) driven by underlying bone marrow failure. ${ }^{6}$ Anemia associated with $\mathrm{PNH}$ is often chronic and can lead to symptoms including shortness of breath, headache and chest pain, as well as an increased risk of morbidity and mortality. ${ }^{23,24}$

An estimated $25 \%-35 \%$ of patients with PNH also experience smooth muscle dystonia, ${ }^{25}$ as free hemoglobin in the serum reacts irreversibly with and depletes nitric oxide (NO), which regulates smooth muscle cells. ${ }^{4,26}$ Low NO levels can cause dyspnea, erectile dysfunction, gastrointestinal spasms, and abdominal pain, as well as pain in other parts of the body. Depletion of NO can also precipitate thrombosis, as it can activate and, thus, cause aggregation of platelets. ${ }^{4}$

Thrombosis occurs in $13.3 \%$ of patients with PNH before treatment initiation and in $40 \%$ of patients overall. ${ }^{9,27}$ Clinical presentation of thrombosis may vary and be difficult to distinguish from general $\mathrm{PNH}$ symptomatology, which includes fatigue, abdominal pain, and chest pain. ${ }^{28}$ In patients with $\mathrm{PNH}$, platelets appear to be more susceptible to terminal complement-induced prothrombin effects, potentially explaining the tendency toward thrombosis. ${ }^{29}$ The most common sites of thrombosis are venous thrombosis of the liver (Budd-Chiari syndrome); abdomen (portal, mesenteric, splenic); and the brain (sagittal and cavernous sinus). The risk of developing thrombosis is correlated with the proportion of $\mathrm{PNH}$ clones and the severity of intravascular hemolysis.,30 Thrombosis is a significant source of morbidity and accounts for up to $50 \%$ of mortality in untreated patients. . $^{40}$ 
Renal failure is a significant complication of $\mathrm{PNH}^{9,26,31}$ Renal failure is likely caused by iron deposits and free plasma hemoglobin, manifesting as acute kidney injury or chronic kidney disease. Chronic kidney disease potentially stems from intravascular hemolysis, while renal tubular damage can occur from microvascular thrombosis. It is estimated that $42.8 \%$ of untreated patients have renal dysfunction, ${ }^{9}$ and it is a significant source of mortality if untreated, contributing to $8 \%-18 \%$ of deaths. ${ }^{31}$

\section{Conclusions}

$\mathrm{PNH}$ is an extremely rare, chronic, life-threatening blood disease with an estimated prevalence of 5,0006,000 in the U.S. population. ${ }^{17,18} \mathrm{PNH}$ is characterized by intravascular and extravascular hemolysis, ${ }^{21}$ leading to severe anemia and other debilitating symptoms. ${ }^{23,24}$ Patients are at risk of fatal disease complications, including thrombotic and hemorrhagic events, ${ }^{9}$ with a 10 -year mortality rate of $29 \%$, if left untreated. ${ }^{14}$

\section{DISCLOSURES}

This research was developed under a research contract between RTI Health Solutions and Apellis Pharmaceuticals and was funded by Apellis Pharmaceuticals. Bektas, Copley-Merriman, and Khan are employees of RTI Health Solutions. Sarda is an employee of Apellis Pharmaceuticals. Shammo consults for Apellis Pharmaceuticals.

\section{ACKNOWLEDGMENTS}

Kate Lothman of RTI Health Solutions provided medical writing services, which were funded by Apellis Pharmaceuticals.

\section{REFERENCES}

1. Janeway CA, Travers P, Walport M, Shlomchik M. Immunobiology: The Immune System in Health and Disease. 5th ed. Garland; 2001.
2. Thau L, Asuka E, Mahajan K. Physiology, opsonization. In: StatPearls. StatPearls Publishing; 2020. Available at: https:// www.ncbi.nlm.nih.gov/books/NBK534215/. Accessed December 14, 2020.

3. Dunkelberger JR, Song WC. Complement and its role in innate and adaptive immune responses. Cell Res. 2010;20(1):34-50.

4. Berentsen S, Hill A, Hill QA, Tvedt THA, Michel M. Novel insights into the treatment of complement-mediated hemolytic anemias. Ther Adv Hematol. 2019;10:2040620719873321.

5. Hill A, DeZern AE, Kinoshita T, Brodsky RA. Paroxysmal nocturnal haemoglobinuria. Nat Rev Dis Primers. 2017;3:17028. 6. Brodsky RA. Paroxysmal nocturnal hemoglobinuria. Blood. 2014;124(18):2804-11.

7. Parker CJ. Update on the diagnosis and management of paroxysmal nocturnal hemoglobinuria. Hematology Am Soc Hematol Educ Program. 2016;2016(1):208-16.

8. Aplastic Anemia and Myelodysplastic Syndromes International Foundation. PNH - paroxysmal nocturnal hemoglobinuria. 2020. Available at: https://www.aamds.org/ diseases/pnh. Accessed December 14, 2020.

9. Schrezenmeier H, Roth A, Araten DJ, Kanakura Y, Larratt L, Shammo JM, et al. Baseline clinical characteristics and disease burden in patients with paroxysmal nocturnal hemoglobinuria (PNH): updated analysis from the International PNH Registry. Ann Hematol. 2020;99(7):1505-14.

10. Parker C, Omine M, Richards S, Nishimura J, Bessler M, Ware R, et al. Diagnosis and management of paroxysmal nocturnal hemoglobinuria. Blood. 2005;106(12):3699-709.

11. de Latour RP, Mary JY, Salanoubat C, Terriou L, Etienne G, Mohty M, et al. Paroxysmal nocturnal hemoglobinuria: natural history of disease subcategories. Blood. 2008;112(8):3099-106.

12. Socie G, Schrezenmeier H, Muus P, Lisukov I, Roth A, Kulasekararaj A, et al. Changing prognosis in paroxysmal nocturnal haemoglobinuria disease subcategories: an analysis of the International PNH Registry. Intern Med J. 2016;46(9):1044-53.

13. Schubert J, Bettelheim P, Brümmendorf TH, Röth A, Schrezenmeier H, Stüssi G. Paroxysmal nocturnal hemoglobinuria (PHN). DGHO Onkopedia. March 2012. Available at: https://www.onkopediaguidelines.info/en/onkopedia/guidelines/ paroxysmal-nocturnal-hemoglobinuriapnh/. Accessed December 19, 2020.
14. Fu R, Li L, Li L, et al. Analysis of clinical characteristics of 92 patients with paroxysmal nocturnal hemoglobinuria: a single institution experience in China. J Clin Lab Anal. 2020;34(1):e23008.

15. Schcolnik-Cabrera A, Labastida-Mercado N, Galindo-Becerra LS, et al. Reduced-intensity stem cell allografting for PNH patients in the eculizumab era: the Mexican experience. Hematology. 2015;20(5):263-66.

16. Mon Pere N, Lenaerts T, Pacheco JM, Dingli D. Evolutionary dynamics of paroxysmal nocturnal hemoglobinuria. PLoS Comput Biol. 2018;14(6):e1006133.

17. Hill A, Platts PJ, Smith A, et al. The incidence and prevalence of paroxysmal nocturnal hemoglobinuria (PNH) and survival of patients in Yorkshire. Blood. 2006;108:985.

18. U.S. Census Bureau. U.S. and world population clock. 2020. Available at: https://www. census.gov/popclock/. Accessed December 14, 2020.

19. Ueda Y, Obara N, Yonemura Y, et al. Effects of eculizumab treatment on quality of life in patients with paroxysmal nocturnal hemoglobinuria in Japan. Int J Hematol. 2018;107(6):656-65.

20. Tenant KF. The FACIT Fatigue Scale (Version 4). 2020. Available at: https://hign. org/consultgeri/try-this-series/facitfatigue-scale-version-4. Accessed December 14, 2020.

21. Brodsky RA. Advances in the diagnosis and therapy of paroxysmal nocturnal hemoglobinuria. Blood Rev. 2008;22(2):65-74.

22. Barcellini W, Fattizzo B. Clinical applications of hemolytic markers in the differential diagnosis and management of hemolytic anemia. Dis Markers. 2015;2015:635670.

23. Harper P, Littlewood T. Anaemia of cancer: impact on patient fatigue and long-term outcome. Oncology. 2005;69(Suppl 2):2-7.

24. Oliva EN, Schey C, Hutchings AS. A review of anemia as a cardiovascular risk factor in patients with myelodysplastic syndromes. Am J Blood Res. 2011;1(2):160-66.

25. Hill A, Rother RP, Hillmen P. Improvement in the symptoms of smooth muscle dystonia during eculizumab therapy in paroxysmal nocturnal hemoglobinuria. Haematologica. 2005;90(12 Suppl):ECR40.

26. Kokoris SI, Gavriilaki E, Miari A, et al. Renal involvement in paroxysmal nocturnal hemoglobinuria: an update on clinical features, pathophysiology and treatment. Hematology. 2018;23(8):558-66. 
27. Borowitz MJ, Craig FE, Digiuseppe JA, et al. Guidelines for the diagnosis and monitoring of paroxysmal nocturnal hemoglobinuria and related disorders by flow cytometry. Cytometry B Clin Cytom. 2010;78(4):211-30.

28. Peacock-Young B, Macrae FL, Newton DJ, Hill A, Ariens RAS. The prothrombotic state in paroxysmal nocturnal hemoglobinuria: a multifaceted source. Haematologica. 2018;103(1):9-17.
29. Wiedmer T, Hall SE, Ortel TL, Kane WH, Rosse WF, Sims PJ. Complement-induced vesiculation and exposure of membrane prothrombinase sites in platelets of paroxysmal nocturnal hemoglobinuria. Blood. 1993;82(4):1192-96.

30. Young NS, Meyers G, Schrezenmeier H, Hillmen P, Hill A. The management of paroxysmal nocturnal hemoglobinuria: recent advances in diagnosis and treatment and new hope for patients. Semin Hematol. 2009;46(1 Suppl 1):S1-S16.
31. Nishimura J, Kanakura Y, Ware RE, et al. Clinical course and flow cytometric analysis of paroxysmal nocturnal hemoglobinuria in the United States and Japan. Medicine (Baltimore). 2004;83(3):193-207.

32. Ricklin D, Mastellos DC, Reis ES, Lambris JD. The renaissance of complement therapeutics. Nat Rev Nephrol. 2018;14 (1):26-47.

\title{
Paroxysmal nocturnal hemoglobinuria: patient journey and burden of disease
}

\author{
Meryem Bektas, PhD, MBA; Catherine Copley-Merriman, MS, MBA; Shahnaz Khan, MPH; \\ Sujata P Sarda, BPharm, PhD; and Jamile M Shammo, MD, FASCP, FACP
}

\section{SUMMARY}

Patients with paroxysmal nocturnal hemoglobinuria $(\mathrm{PNH})$ often experience a lengthy path to diagnosis. Fewer than $40 \%$ of patients with $\mathrm{PNH}$ receive a diagnosis within 12 months of symptom onset, and $24 \%$ of all PNH diagnoses can take 5 years or longer. Diagnostic delay is a source of distress and can affect emotional well-being for patients with $\mathrm{PNH}$. In $\mathrm{PNH}$ disease management, patients and care providers focus on risk of organ failure and mortality related to disease progression; nonetheless, patients' healthrelated quality of life (HRQOL) is largely affected by extensive treatment requirements and nonfatal complications of disease, such as fatigue. In particular, thrombosis is associated with significant impairments in physical and social functioning and global health status and significant fatigue. Among patients with anemia who are transfusion dependent, the burden of transfusion is considerable. Transfusion dependence has a negative effect on HRQOL; is associated with risks and complications, including iron overload; and results in lost productivity due to travel times to and time spent at infusion centers.

\section{Author affiliations}

Meryem Bektas, PhD, MBA; Catherine Copley-Merriman, MS, MBA; and Shahnaz Khan, MPH, Market Access and Outcomes Strategy, RTI Health Solutions, Research Triangle, NC. Sujata P Sarda, BPharm, PhD, Global Health Economics and Outcomes Research, Apellis Pharmaceuticals, Waltham, MA, and Jamile M Shammo, MD, FASCP,

FACP, Department of Internal Medicine, Rush University Medical Center, Chicago, IL.

\section{AUTHOR CORRESPONDENCE: \\ Meryem Bektas, 919.541.6776; \\ mbektas@rti.org}

